\title{
Transition Energy, Orientation Force and Work Done in Transitional Behavior Atoms: Formulating New Principles in Thermodynamics
}

\author{
Mubarak Ali \\ Department of Physics, COMSATS University Islamabad, Islamabad Campus, Park \\ Road, 45550, Pakistan, E-mail: mubarak74@mail.com, mubarak74@comsats.edu.pk
}

\begin{abstract}
A study of different parameters in thermodynamics is important to explore the science of various phenomena. Solid atoms are related to the science of condensed matter when their transition states do not reinstate into the original states. The same is the case with gaseous atoms but in a different way. An anomaly in the first law of thermodynamics can be found while studying transitional behaviors of atoms. A gaseous atom involves transitional energy in a gaining manner while undertaking transition state. Hence, the work is carried out by that gaseous atom. In fact, this should be registered symbolically in a plus form. A solid atom involves transitional energy absorbed in undertaking transition state. Hence, the work is carried out on that solid atom. In fact, this should be registered symbolically in a minus form. Thus, anomaly is resolved for equations of change in internal energy of the system. The transition energy introduces different transition states in the system which is composed of gaseous or solid atoms. Hence, gaseous and solid atoms engage different orientation forces to orientate their electrons. In an atom, transition energy changes potential energy of an electron, whereby it controls the position through orientation force. Gaseous and solid atoms introduce cooling and heating effects when electrons start to restore from the mid-states. In gaseous or solid atom, a mid-state exists between re-crystallization and liquid states. An electron executes dynamics by remaining within the occupied energy knot. Thus, nonstop elastically-driven electronic states of atoms are the cause of entropy and irreversible cycle.
\end{abstract}

Keywords: Transition energy; Orientation force; Work done; Internal energy; Cooling and Heating; Entropy 


\section{Introduction}

A careful observation of atomic behavior with respect to influencing force at the electron level shows irregularity in the basic principles of thermodynamics. The anomaly is explicitly found in the first law of thermodynamics. The law is one of the essential elements of thermodynamics, which governs the science behind many remarkable applications.

Originally, the work was taken negatively when it was performed by gaseous atoms under compression; again, the work was taken positively when it was performed on gaseous atoms under expansion. Contrarily, this study handles the aforementioned issue in an entirely different manner. The work performed by solid atoms can be differentiated, and the work performed on solid atoms can also be differentiated.

The exerting force at the electron level in gaseous atoms does not express the relation with change in pressure, temperature and volume. The force per unit area is a particularized pressure only. This study deals with the exerting force at electron level in levitational manner for the system composed of gaseous atoms, and in gravitational manner for the system composed of solid atoms. Hence, the change in internal energy of system comprising atoms of gaseous state or solid state interprets the law in different ways.

Atoms of gaseous and solid states while undertaking liquid states deal with different force and energy relationship [1]. The phenomena of heat and photon energy while dealing with matter at atomic and electronic levels were discussed in a separate study [2]. The flowing atoms of inert behavior split under the propagation of excessive photonic field [3]. In certain cases, where electrons of atoms do not entertain the modes of conservative forces, atoms form a structure rather than evolve a structure [4]. Atoms, in which electrons execute interstate dynamics under the conservative forces, evolve structures rather than develop structures [5].

Considering the formation of tiny-sized particles and their coalescences for the large-sized particles, a preliminary study on the origin of physics and chemistry was discussed earlier [6]. Controlling the morphology and structure of gold tiny particles was mainly due to the tuned pulse rates [7]. The role of atoms in tiny-sized particles is critical 
while using them for nanomedicine application [8]. Due to different natures of carbon atoms, tiny grain carbon films deal with different behaviours under the analyses [9].

Assembling blocks for larger particles provides a possibility for treating atoms and molecules as materials in future [10]. Understanding the dynamics of formation of tiny particles is essential prior to the study of assembly into a larger particle [11]. Under the identical process conditions, gold and silver precursors were processed to develop different shaped tiny-sized particles and larger-sized particles [12]. Under the suitable transition of atoms, TiN coating was deposited on the substrate by employing the physical vapor deposition system [13].

This study describes mathematically a distinct nature of the force under transitional energy of the gaseous atom and the solid atom. The gaseous atom formulates different equations of work when it is carried out on the atom as well as carried out by the atom. Hence a system, composed of gaseous atoms, formulates different equations of change in internal energy. A solid atom also formulates different equations of work when it is carried out on the atom as well as carried out by the atom. Thus a system, composed of solid atoms, formulates different equations of change in internal energy.

The transition energy introduces the mid-states of electrons of both gaseous and solid atoms near the ground surface. When the electron of gaseous atom restores its attained mid-state to the original state, a process of cooling is observed. When the electron of solid atom restores its attained mid-state to the original state, a process of heating is observed.

\section{Results and Discussion}

According to the existing principles of thermodynamics, the internal energy of a gas system varies mainly due to the temperature. If the internal energy of the gaseous atoms varies by varying the temperature, then almost the same can apply to solid atoms. However, a different nature of transition in solid atom and gaseous atom signifies a different scope of physical or chemical science.

In the atoms of gaseous and solid states, the liquid states at appropriate level of ground surface are accessible. In both gaseous atoms and solid atoms, the access to 
liquid state is under the suitable force and energy relationship [1]. Hence, a relation between force and energy of the atom is directly related to occupied electrons.

\subsection{Condensed Matter Science}

When it is said that an atom is working, it becomes meaningful as the atom is in the state of transition. The scheme of force and energy varies for it in the course of breaking ideal isolation. Hence, the first law of thermodynamics needs to be addressed in this perspective. In the processing of different solid materials, it is hard to say that atoms retain the original states. This points out that solid atoms also undertake elongation and deformation. Elongation and deformation of solid atoms fall under the condensed matter science.

In dead, solid atoms undertake different transition states. Solid atoms, when found in the condensed states, had adopted plastically-driven electronic states. Atoms have been solidified. Atoms can no longer deal with the elastically-driven electronic states, so solid atoms are in different frozen states. Such frozen states of the solid atoms are resulted under the deforming and elongating behaviors.

Gaseous atoms can also give birth to condensed matter science as they deal with transitions, too. However, gaseous atoms mainly deal with the squeezing behaviors. Due to the different behavior of gaseous atoms as compared to solid atoms, condensed matter science can be expressed in a different way. Therefore, gaseous atoms and solid atoms introduce different condensed matter science. In the physical behavior of condensed matter science, the study of physical sciences should furnish and grow. In the chemical behavior of condensed matter science, the study of chemical sciences should furnish and grow.

\subsection{Transition Energy vs. Orientation Force}

In the transitional behavior of atoms, levitational force and gravitational force are orientating electrons. The transition states of atoms remain successively in recovery, neutral, re-crystallization and liquid states [1]. However, the aim of this study is to explore the transition state called mid-state. The mid-state exists between recrystallization state and liquid state. The mid-state of the atom or the mid-state of the 
under-study electron is either before the liquid state or after the re-crystallization state, so the mid-state can be said to be an 'attaining liquid state'.

In an atom, potential energy of the electrons under involved energy change the trend of energy-knot-net [3]. Hence, an orientation force of the electrons should vary. In this way, gaseous atoms or solid atoms can deal with different orientation force and different potential energy of the electrons to reveal different trends of the energy-knot-nets.

In each transition state of an atom having gaseous state or having solid state, transitional energy (ET) of different levels is involved to keep the required potential energy of the electrons. Therefore, electrons deal with the prepared conditions of the occupied energy knots. On involving ' $\mathrm{E}_{\mathrm{T}}$ ' of an atom, the force is engaged to tilt electrons in the required orientation. However, a fixed orientation force to each electron is being involved in the original state of an atom. In this manner, electrons keep the conserved energy of their atom instead of ' $E T$ '. Under normal conditions, an atom cannot break the ideal isolation; therefore, it keeps ground point in the original format of exerting force. In the course of transition, an atom is with some more or some less force and energy.

Electrons of transitional behavior atoms undertake infinitesimal displacements where their energy knots also remain occupied. In the transitional behavior atoms, such transitions of electrons do not infer their migration from filled states to nearby unfilled states. In other words, atoms do not execute confined interstate electron dynamics when their electrons undertake infinitesimal displacements. The transitions of gaseous or solid atoms are under the variation of orientation force and potential energy of their electrons. Hence, the energy required to introduce the transition of an atom is referred to the transition energy. The occupied energy knots to electrons undertake stretching in the solid atom and contraction in the gaseous atom.

Atoms of different gaseous states are basically categorized with respect to the nature-granted characteristics. The same applies to atoms of solid states. In the original state, atom of any element deals with the conserved energy and force. According to transitional behavior of the atom, electrons tilt for different orientations by remaining within the occupied energy knots. Electrons of gaseous atoms deal with the levitational force $(F\llcorner)$ and electrons of solid atoms deal with the gravitational force $(F G)$. So, 
gaseous and solid atoms keep different levels (or different amounts) of 'ET'. A different level (or a different amount) of ' $\mathrm{E}_{T}$ ' also remains within the atoms of gaseous state or solid state. However, force and energy for each transition state of the atom, either in gaseous state or in solid state, varies at the minute level. Nonetheless, atoms exhibit different features when dealing with sizes at microlevels to nano levels.

Energy, which introduced a transition in the state of gaseous atom or in the state of solid atom, is termed as transition energy. The introduced behavior of transition energy is related to 'ET'. Force, which introduced a change in the orientation of electron of gaseous atom or of electron of solid atom, is termed as orientation force. The introduced behavior of orientation force is related to the orientational force. In the gaseous atom, the introduced behavior of orientation force is due to ' $F_{L}$ '. In the solid atom, the introduced behavior of orientation force is due to ' $\mathrm{F}_{\mathrm{G}} \mathrm{G}$.' When a gaseous atom gains ' $\mathrm{ET}_{\mathrm{T}}$ ' to attain the mid-state, ' $\mathrm{FL}$ ' of electrons decreases while potential energy of electrons increases. When a solid atom absorbs ' $\mathrm{ET}_{\mathrm{T}}$ ' to attain the mid-state, both ' $\mathrm{F}_{\mathrm{G}}$ ' and potential energy of electrons decrease.

\subsection{Work Done in Atoms}

To decrease ' $F L$ ' of a gaseous atom at the electron level, ' $\mathrm{E}_{\mathrm{T}}$ ' is gained by it. The gained 'ET' of a gaseous atom keeps maintaining potential energy of left-positioned electrons and right-positioned electrons while undertaking clockwise and anti-clockwise tilting, respectively. For transitional behavior of a gaseous atom, electrons deal with the infinitesimal displacements by engaging the ' $F L$ ', where they remain within the occupied energy knots. The job is carried out by gaseous atom to convert it into the mid-state. Thus, gaseous atom works positively to change the state as given in equation (1).

$\Delta \mathrm{W}=+\mathrm{F}_{\mathrm{L}}(\mathrm{dx} \perp)$

However, attained mid-state of gaseous atom undergoes the process to reinstate the original gaseous state. To reinstate the original gaseous state is not done by atom itself. (To be in elastically-driven electronic state, transitional behavior gaseous atom apparently requires an external environment, source or regulator.) Hence, the work is 
carried out on the atom of attained mid-state to reinstate it into the original state as given in equation (2).

$\Delta \mathrm{W}=-\mathrm{F}_{\mathrm{L}}\left(\mathrm{dx}_{\mathrm{T}}\right) \ldots(2)$

In equations (1) and (2), $\Delta \mathrm{W}$ designates 'change in work'. In equation (1), ( $\mathrm{dx} \perp$ ) indicates the infinitesimal displacements of electrons from top-to-bottom. In equation (2), $\left(\mathrm{dx}_{\mathrm{T}}\right)$ indicates the infinitesimal displacements of electrons from bottom-to-top.

Many gaseous atoms can keep ground points near the ground surface when they attain the mid-states. Figure 1 (a) shows the hypothesized gaseous atom when it is in original gaseous state and when it is in attained mid-state. The position of hypothesized gaseous atom when in the original state is above the suitable level of ground surface.

Figure 1: (a) Position of the
gaseous atom under original
state (1) and under attained mid-
state (2), and suitable level of
ground surface (3). (b) Position
of the solid atom under original
state (1) and under attained mid-
state (2), and suitable level of
ground surface (3)

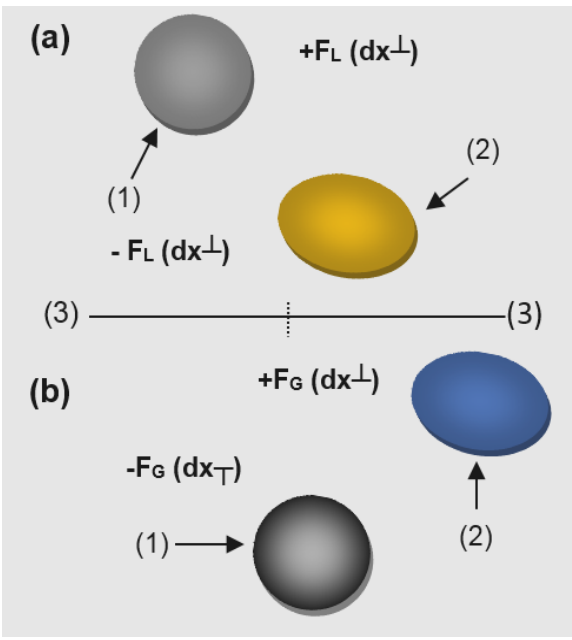

A solid atom absorbs ' $\mathrm{E}_{T}$ ' to decrease ' $\mathrm{F}_{\mathrm{G}}$ ' at the electron level. The absorbed 'ET' of solid atom keeps maintaining the potential energy of left-positioned electrons and rightpositioned electrons while undertaking their anti-clockwise and clockwise tilting, respectively. For transitional behavior of a solid atom, electrons deal with infinitesimal displacements by engaging ' $F_{G}$ ', where they remain within the occupied energy knots. The job is carried out on the gaseous atom to convert it into the mid-state. Thus, solid atom works negatively to change the state as given in equation (3).

$\Delta \mathrm{W}=-\mathrm{F}_{\mathrm{G}}\left(\mathrm{dx_{T }}\right) \ldots(3)$ 
In equation (3), $\left(\mathrm{dx}_{\mathrm{T}}\right)$ indicates the infinitesimal displacements of electrons from bottom-to-top. $\Delta \mathrm{W}$ designates 'change in work'.

But, the resultant (mid-state) atom works itself in restoring the original state, i.e., the solid state. Hence, the atom of attained mid-state reinstates (self) into the original state as given in positive form in equation (4). (To be in elastically-driven electronic state, transitional behavior solid atom does not require an external environment, source or regulator.)

$\Delta \mathrm{W}=+\mathrm{FG}_{\mathrm{G}}\left(\mathrm{dx} \mathrm{X}^{\perp}\right) \ldots(4)$

In equation (4), $\left(d^{\perp} \perp\right)$ indicates the infinitesimal displacements of electrons from topto-bottom. $\Delta \mathrm{W}$ designates 'change in work'.

Many solid atoms can keep ground points near the ground surface when they attain the mid-states. Figure 1 (b) shows the hypothesized solid atom when it is in original solid state and when it is in attained mid-state. The position of hypothesized solid atom when in the original solid state is below the suitable level of ground surface.

\subsection{Original States vs. Mid-States of Atoms}

Gaseous atoms keep the approximate orientation of electrons $40^{\circ}$ along the north pole in original state [1]; orientation is on the left sides to normal lines drawn from the centers in the left-positioned electrons, and orientation is on the right sides to normal lines drawn from the centers in the right-positioned electrons.

When a suitable gaseous atom undertakes transition state, it gains energy, i.e., tilting of electrons is downward from the original positions. The involved ' $\mathrm{E}_{\mathrm{T}}$ ' of an atom engages ' $\mathrm{FL}_{\mathrm{L}}$ to orientate the electrons. While, tilting of electrons (of gaseous atom) to attain the mid-state, ' $\mathrm{E}_{\mathrm{T}}$ ' is involved in the sense of gaining. An orientated position of electron of the hypothesized gaseous atom is shown in Figure 2 (a). (However, to reinstate original gaseous state of an atom from the mid-state, ' $\mathrm{ET}^{\mathrm{T}}$ ' is involved in the sense of absorbing.)

Solid atoms keep the approximate orientation of electrons $40^{\circ}$ along the south pole in original state [1]; orientation is on the left sides to normal lines drawn from the centers 
in the left-positioned electrons, and orientation is on the right sides to normal lines drawn from the centers in the right-positioned electrons.

When a suitable solid atom undertakes transition state, it absorbs energy, i.e., tilting of electrons is upward from the original positions. The involved ' $\mathrm{E}_{\mathrm{T}}$ ' of an atom engages the ' $F_{G}$ ' to orientate the electrons. While tilting of electrons (of solid atom) to attain the mid-state, 'ET' is involved in the sense of absorbing. In this case, a single electron of the hypothesized solid atom is shown in Figure 2 (b). (However, to reinstate original solid state of an atom from the mid-state, ' $\mathrm{E}_{\mathrm{T}}$ ' is involved in the sense of gaining.)

Figure 2: (a) A single electron of the hypothesized gaseous atom: (1) original gaseous state; (2) transitional behavior gaseous state. (b) A single electron of the hypothesized solid atom: (1) original solid state; (2) transitional behavior solid state
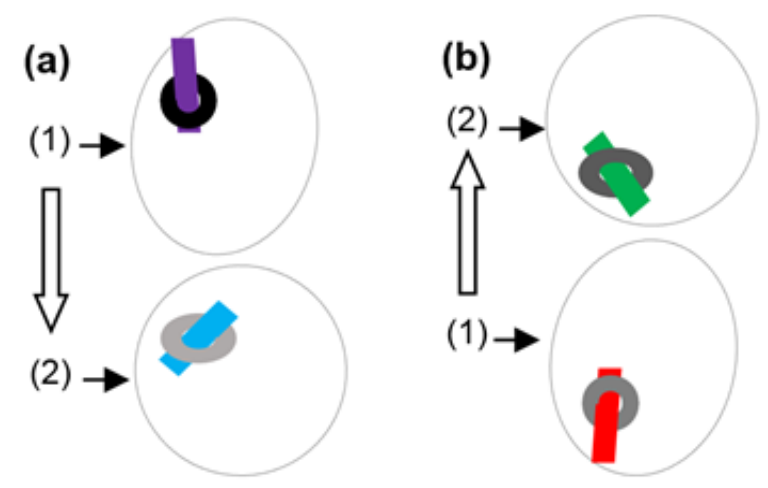

\subsection{Internal Energy of Composed System Atoms}

According to the existing principles of thermodynamics, a change occurs in the internal energy (dU) of a gaseous atom. Moreover, when it is converted into the mid-state, it is stated as $d U=\Delta Q-\Delta W$. On substituting the value of the investigated work as shown in equation (1), the equation becomes $d U=\Delta Q-F L(d x \perp) \ldots$ (5). Here, ' $\Delta Q$ ' is related to change in ' $\mathrm{E}^{\mathrm{T}}$ ', which is in a gained manner.

However, a change in the internal energy (dU) of gaseous atom occurs when it is restoring from the attained mid-state. It is stated as $d U=\Delta Q-(-\Delta W)$. On substituting the value of the investigated work as shown in equation (2), the equation becomes $\mathrm{dU}=\Delta \mathrm{Q}$ $+F_{L}\left(d_{T} x_{T}\right) \ldots(6)$. Here, ' $\Delta Q$ ' is related to 'ET', which is in an absorbed manner.

A change in the internal energy (dU) of solid atom occurs when it is converted into the mid-state. It is stated as $d U=\Delta Q-(-\Delta W)$. After substituting the value of the investigated work as shown in equation (3), the equation becomes $d U=\Delta Q+F_{G}\left(d x_{T}\right)$ ... (7). Here, $\Delta Q$ is related to ' $E T$ ', which is in an absorbed manner. 
A change in the internal energy (dU) of solid atom occurs when it is restoring from the attained mid-state. It is stated as $\mathrm{dU}=\Delta \mathrm{Q}-\Delta \mathrm{W}$. On substituting the value of the investigated work as shown in equation (4), the equation becomes $d U=\Delta Q-F_{G}(d x \perp)$ ... (8). Here, $\Delta \mathrm{Q}$ is related to ' $\mathrm{E} T$ ', which is in a gained manner.

\subsection{Cooling Effect of Gaseous Atoms}

When the left-positioned electron to the centre of hypothesized gaseous atom is considered to attain the mid-state, typical energy shaped like 'downward arc ends' ( $\neg$ ) is generated in the clockwise tilting (of the electron). A typical energy shaped like 'downward arc ends' $\left({ }^{\frown}\right)$ is shown in Figure $3($ a).
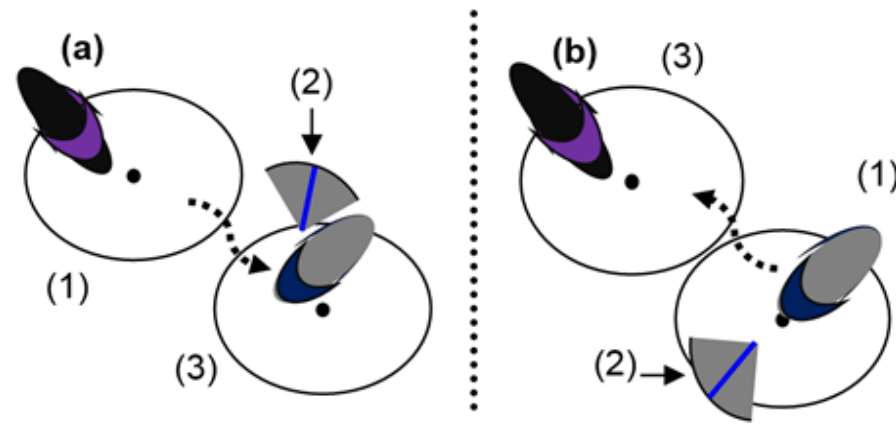

Figure 3: (a) An arc-shaped typical energy is generated along the clockwise tilting of left-positioned electron (of hypothesized gaseous atom) when converting gaseous state into the mid-state, where orientation under the exerting force is $40^{\circ}$ to $5^{\circ}$ left, under the impartial force is $5^{\circ}$ to $0^{\circ}$ left and under the exerting force is $0^{\circ}$ to $40^{\circ}$ right from the normal line drawn to the centre: (1) gaseous state electron; (2) typical energy shaped

like 'downward arc ends'; (3) attained mid-state gaseous electron. (b) Removal of typical energy of cooling when it is restoring the attained mid-state to gaseous state under self-driven orientation and potential energy of electron $\left(40^{\circ}\right.$ to $0^{\circ}$ right, $0^{\circ}$ to $5^{\circ}$ left and $5^{\circ}$ to $40^{\circ}$ left): (1) attained mid-state gaseous electron; (2) removal of typical energy; (3) gaseous state electron

In the hypothesized gaseous atom where left-positioned electron is considered, the orientation of the tilting electron under the exerting force is along the $40^{\circ}$ to $5^{\circ}$ left, under the impartial force is along the $5^{\circ}$ to $0^{\circ}$ left and under the exerting force is along the $0^{\circ}$ to $40^{\circ}$ right. These different orientations are from the normal lines drawn to the centre of 
electron under-study. Hence, left-positioned electron plots the trajectory of force and energy shaped like 'downward arc ends'.

When the attained mid-state electron starts to restore the original gaseous state by anti-clockwise tilting, typical energy is removed from the point of generation. Upon separation, a generated typical energy is related to cooling. In gaseous atom, removal of typical energy follows an eccentric behavior, where it inverts the shape as shown in Figure 3 (b). The round side of arc-shaped typical energy causes the cooling effect.

\subsection{Heating Effect of Solid Atoms}

When the left-positioned electron to the centre of hypothesized solid atom is considered to attain the mid-state, typical energy shaped like 'upward arc ends' ( $\smile$ ) is generated in the anti-clockwise orientation. A typical energy shaped like 'upward arc ends' is shown in Figure 4 (a).

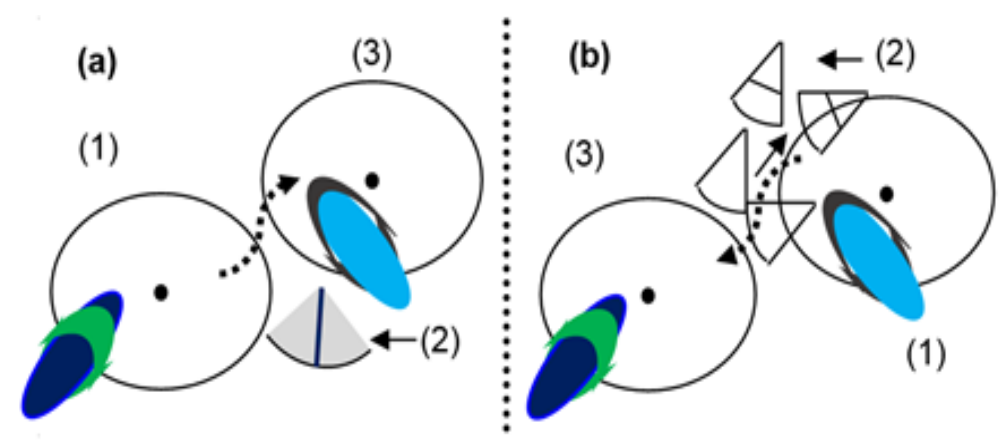

Figure 4: (a) An arc-shaped typical energy is generated along the anti-clockwise tilting of left-positioned electron (of hypothesized solid atom) when it is converting solid state into the attained mid-state, where orientation under the exerting force is $40^{\circ}$ to $5^{\circ}$ left, under the impartial force is $5^{\circ}$ to $0^{\circ}$ left and under the exerting force is $0^{\circ}$ to $40^{\circ}$ right from the normal line drawn to the centre: (1) solid state electron; (2) typical energy shaped like 'upward arc ends'; (3) attained mid-state solid electron. (b) Removal of typical energy in the form of tits and bits of heat when it is restoring the attained midstate to solid state under self-driven orientation and potential energy of electron ( $40^{\circ}$ to $0^{\circ}$ right, $0^{\circ}$ to $5^{\circ}$ left and $5^{\circ}$ to $40^{\circ}$ left): (1) attained mid-state solid electron; (2) removal of typical energy; (3) solid state electron 
In the hypothesized solid atom, where left-positioned electron is considered, the orientation of the tilting electron under the exerting force is along the $40^{\circ}$ to $5^{\circ}$ left. Under the impartial force, it is along the $5^{\circ}$ to $0^{\circ}$ left, and under the exerting force, it is along the $0^{\circ}$ to $40^{\circ}$ right. These different orientations are from the normal lines drawn to the centre of electron, which configure the trajectory of typical energy shaped like 'upward arc ends'. The typical energy is in the same shape as in the case of electron of gaseous atom under study. However, the typical energy is generated by the understudy electron when it orientates along the south pole.

When an electron restores an attained mid-state to the solid state, the removal of typical energy at the point of generation is not due to the eccentricity. Typical energy shaped like 'upward arc ends' tilts to opposite side from the point of generation. On tilting typical energy shaped like 'upward arc ends', it divides into tits and bits of heat as shown in Figure 4 (b). The same is the case with right-positioned electron to the centre of solid atom when it was considered to execute dynamics.

Despite clockwise tilting of the electron, the orientation of electron is still along the south pole. Thus, the process of heating is observed in both left and right sided electrons of the hypothesized solid atom when they are restoring the attained mid-states to original states. The tips of divided tits and bits (from arc-shaped typical energy) cause heating effect.

\subsection{Nonstop Elastically-driven Electronic States and Entropy}

In two different kinds of typical energy, the nature of both force and energy also becomes different. Restoring original positions of electrons within their occupied energy knots, electrons tilt under the self-driven orientation force. Electrons keep their potential energy as per scheme of their occupied energy knots in gaseous atom or in solid atom. Electrons of respective gaseous atom and solid atom preserve their timing to meet the exerting force in cooling and heating processes. Solid and gaseous atoms do not actually modify the shapes. Gaseous and solid atoms deal with the nonstop elasticallydriven electronic states to generate respective cooling and heating effects. Electrons deal with the recoverable contraction of occupied energy knots in a gaseous atom. On 
the other hand, electrons deal with the recoverable stretch of occupied energy knots in a solid atom.

In both gaseous and solid atoms, where electron dynamics do not meet with the element of force, only energy remains involved. Hence, the force remains impartial in the zone, where electron dynamics meet only with energy. At this point, a disorder between force and energy has been developed. Hence, the issue of entropy can relate to the zone of impartial force. To get $100 \%$ efficiency as described by the Carnot's Engine, a discussion on the entropy can be further investigated from electron to atom. Hence, the discussion on entropy will also branch into nano-sized and micro-sized components.

Entropy develops the understanding in artistic manner when the study of atoms deals with the orientation at electron level. More specifically, entropy develops understanding for atoms of such elements, which execute the nonstop elastically-driven electronic states. In the only elastically-driven electronic states, i.e., when atoms only deal with the elastic behavior rather than nonstop elastic behavior, entropy should not be a big study as it can be the subject of minor details. So, entropy is not a considerable aspect when studying atoms for their elastically-driven electronic states. However, entropy can be the major subject in the case of plastically-driven electronic states of the atoms. In solid atoms, in addition to studying the entropy with major detail, the study of condensed matter science is also there, where elongation and deformation processes are the core subjects. In gaseous atoms, in addition to studying the entropy with major detail, the study of condensed matter science is also necessary, where the process of squeezing is the core subject.

While dealing with orientation $0^{\circ}$ to $\sim 5^{\circ}$ of electron executing dynamics, a portion of only fused energy (in the generated typical energy) is due to the existing zone of impartial force. Thus, energy remains fused (merged) only in that zone. So, the orientation of electron is vital to attain the preservation of energy and force. Hence, an electron is the smallest source, which can keep energy and force under preservation.

These kinds of studies enable one to study irreversible cycle and other related topics available in the thermodynamics. An orientation of electron in atoms while executing 
dynamics is also a cause of irreversible cycle. This indicates that different parameters in thermodynamics require re-investigations.

\subsection{General Discussion}

A degree of hotness or a degree of coldness of the system is composed of atoms, and it is related to its transition energy, so a transition energy relates to a temperature of system. Under the suitable transition state, gaseous atoms formed by a particular system can deal with temperature in a few degrees of coldness to many degrees of coldness (minus sign). Under the suitable transition state, solid atoms are composed of a particular system, and they can deal with temperature in a few degrees of hotness to many degrees of hotness (plus sign).

Tits and bits are the broken pieces of the bit energy. Tits and bits are the results of division of 'unit photon energy'. When tits and bits of heat energy convert into arc shaped typical energy, this is by wrapping the element of force shaping along the electron executed dynamics. An electron executes dynamics by remaining within the occupied energy knot. A unit photon gives energy of two bits, and a photon of the minimum length gives energy of four bits [2]. In tits and bits, the element of force has been eliminated. However, this is not the case for a unit photon or an overt photon. The definition of change in energy $(\Delta Q)$ of atoms is related to both heating and cooling, so the usage of ' $\mathrm{mc} \Delta \mathrm{T}$ ' equation should also be addressed accordingly.

Atomically precise gold nanoclusters having pre-determined sizes and structures could be possible under the detailed analyses of varying temperature in synthesis [14]. An exertion of force in the amalgamation of nanoscale and micron-sized components is discussed [15]. Due to different forces exerting to the electrons of atoms of tiny-shaped particles, they develop particles of different geometrical shapes [16]. Atoms at work reveal charge dynamics [17]. To describe the structure, on-going research activities should also consider dynamics in addition to entropy and geometry [18]. However, according to this study, nanoscale and micron-sized components hide several new insights, where not only entropy, geometry and dynamics are required to express structure but orientation, too. 


\section{Conclusion}

Solid atoms do not recover the transition states; they can relate to the condensed matter science. This way, gaseous atoms also do not recover the transition states; they can also deal with the condensed matter science. However, in order to attain mid-state of a gaseous atom, the transition energy involves in a gaining manner. So, electrons tilt downward, where transition energy engages ' $\mathrm{FL}^{\prime}$ ' to control the orientations (of tilting electrons). To attain mid-state of a solid atom, the transition energy involves in an absorbing manner. So, electrons tilt upward, where transition energy engages ' $F_{G}$ ' to control the orientations (of tilting electrons).

When a gaseous atom undergoes for transitional behavior by gaining transition energy, it deals with the accomplished work by itself. However, when a solid atom undergoes for transitional behavior by absorbing transition energy, it does not deal with the accomplished work by itself. A different execution of work in gaseous and solid atoms opens the new windows for research.

In an equation of gaseous atoms when attaining mid-states and in the equation of gaseous atoms when restoring mid-states, the force of levity controls the orientations of electrons. And it is because of the introduced transition energy. Gaseous atoms formulate different equations of change in internal energy in the attaining-mid-state and in the restoring-mid-state. In an equation of solid atoms when attaining mid-states and in the equation of solid atoms when restoring mid-states, the force of gravity controls the orientations of electrons. And it is because of the introduced transition energy. Solid atoms also formulate different equations of change in internal energy in the attainingmid-state and in the restoring-mid-state. Derived equations of change in internal energy open the new areas of research both in theory and experiment.

On decreasing the ' $\mathrm{LL}_{\mathrm{L}}$ ' of electrons, gaseous atoms attain mid-state when the leftpositioned electrons tilt to the right-clockwise under the controlled orientation, and the right-positioned electrons tilt to the left-anti-clockwise under the controlled orientation. Tilting of the electrons is from the north sides. On decreasing the ' $F_{G}$ ' of electrons, solid atoms attain their mid-states where the left-positioned electrons tilt to the right-anticlockwise under the controlled orientation, and the right-positioned electrons tilt to the left-clockwise under the controlled orientation. Tilting of the electrons is from the south 
sides. (A mid-state of atom is prior to liquid state, where an electron keeps an approximate orientation of $40^{\circ}$ instead of $50^{\circ}$.)

Having attained the mid-state, an electron generates typical energy shaped like 'downward arc ends'. When that electron starts restoring the mid-state to the original (gaseous) state, it gives away the generated typical energy in the cooling form. The removal of typical energy of cooling is due to the eccentricity, where the shape like 'downward arc ends' is sustained. In case of solid atom, when that electron starts restoring the mid-state to the original (solid) state, it gives away the generated typical energy in the heating form. The removal of typical energy of heating is due to the conventionality. Having removed, typical energy shaped like 'upward arc ends' divides into tits and bits of heat.

To generate typical energy of cooling and heating, gaseous and solid atoms execute nonstop elastically-driven electronic states. Orientation force and potential energy of the electrons self-restore. So, their mid-states reinstate to the original states. Orientation force and potential energy are within the permissible limit of contraction of energy knots clamped electrons in gaseous atom. Orientation force and potential energy are within the permissible limit of stretching of energy knots clamped electrons in solid atom. Therefore, for studying structure in different materials, it is vital to consider the role of electron orientation.

The study opens doors to address the issue of entropy in different areas. The research on the typical energy generated under the controlled orientations of electrons in both gaseous and solid atoms can play a central role in the current challenges in energy, health and environment sectors. Thus studies of atoms, molecules and their clusters open up new horizons to science.

\section{References}

1. M. Ali, Atoms in Gaseous and Solid States and their Energy and Force Relationships under Transitional Behaviors. (2020), https://doi.org/10.21203/rs.3.rs-88120/v2

2. M. Ali, Heat and Photon Energy Phenomena: Dealing with Matter at Atomic and Electronic Level. https://www.preprints.org/manuscript/201701.0028/v10 
3. M. Ali, Atoms of None of the Elements lonize While Atoms of Inert Behavior Split by Photonic Current. (2020), http://arxiv.org/abs/1611.05392 (last version)

4. M. Ali, Atomic Structure and Binding of Carbon Atoms. https://www.preprints.org/manuscript/201801.0036/v10

5. M. Ali, Structure Evolutions in Atoms of the Elements Executing Confined Interstate Electron Dynamics. (2020), http://arxiv.org/abs/1611.01255 (last version)

6. M. Ali, I $-\mathrm{N}$. Lin, Formation of tiny particles and their extended shapes: origin of physics and chemistry of materials. Appl. Nanosci. 9 (2019) 1367-1382.

7. M. Ali, I-N. Lin, Controlling morphology-structure of gold tiny particles, nanoparticles and particles at different pulse rates and pulse polarity. Adv. Nat. Sci.: Nanosci. Nanotechnol. 10 (2019) 025015.

8. M. Ali, Nanoparticles-Photons: Effective or Defective Nanomedicine. J. Nanomed. Res. 5 (2017) 00139.

9. M. Ali, I -N. Lin, Phase transitions and critical phenomena of tiny grains carbon films synthesized in microwave-based vapor deposition system. Surf. Interface Anal. 51 (2019) 389-399.

10.S. C. Glotzer, M. J. Solomon, Anisotropy of building blocks and their assembly into complex structures, Nature Mater. 6 (2007) 557-562.

11.S. Link, M. A. El-Sayed, Shape and size dependence of radiative, nonradiative and photothermal properties of gold nanocrystals. Int. Rev. Phys. Chem. 19 (2000) 409453.

12. M. Ali, I -N. Lin, C. -J. Yeh. Tapping Opportunity of Tiny-Shaped Particles and Role of Precursor in Developing Shaped Particles. NANO 13 (7) (2018) 1850073 (16 pages).

13. M. Ali, E. Hamzah, M. R. M. Toff. Hard coating deposits: incompatible working energy and forced behaviors of gaseous and solid atoms. Adv. Mater. Process. Technol. (2020), https://doi.org/10.1080/2374068X.2020.1822055

14. T. Chen, Q. Yao, X. Yuan, R. R. Nasaruddin, J. Xie. Heating or Cooling: Temperature Effects on the Synthesis of Atomically Precise Gold Nanoclusters. J. Phys. Chem. C 121 (20) (2017) 10743-10751. 
15. M. Ali, I -N. Lin. Forcing or Forced Exertions in Amalgamation of Nanoparticles and Particles inside the Solution. (2020) ChemRxiv, Preprint, https://doi.org/10.26434/chemrxiv.9823625 (last version)

16. M. Ali, I -N. Lin. Gold Nanostructures and Microstructures with Tunable Aspect Ratios for High-Speed Uni- and Multidirectional Photonic Applications. ACS Appl. Nano Mater. 3 (9) (2020) 9410-9424.

17. C. Kisielowski. Observing Atoms at Work by Controlling Beam-Sample Interactions. Adv. Mater. 27 (2015) 5838-5844.

18. V. N. Manoharan. Colloidal matter: Packing, geometry, and entropy. Science 349 (2015) 1253751.

\section{Author's biography:}

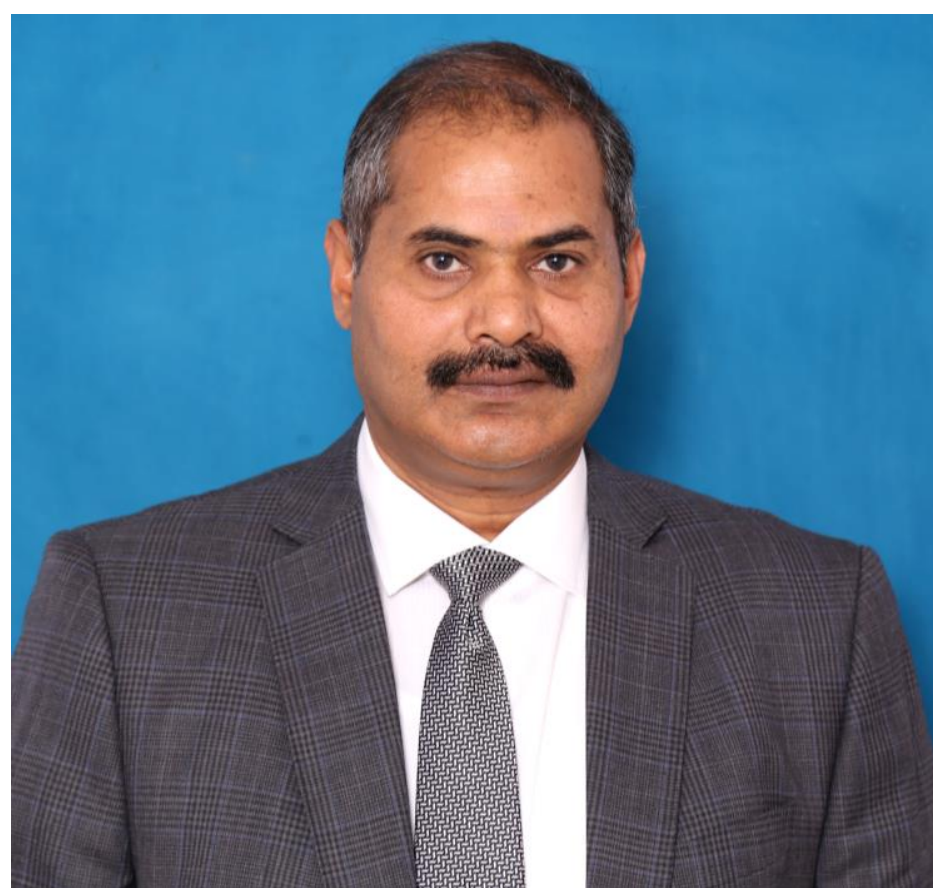

Mubarak Ali graduated from University of the Punjab with BSc (Phys \& Maths) in 1996 and MSc Materials Science with distinction from the Bahauddin Zakariya University, Multan, Pakistan (1998); his thesis work completed at Quaid-i-Azam University Islamabad. He gained PhD in Mechanical Engineering from the Universiti Teknologi Malaysia under the award of Malaysian Technical Cooperation Programme (MTCP;2004-07) and postdoc in advanced surface technologies at Istanbul Technical University under the foreign fellowship of The Scientific and Technological Research Council of Turkey (TÜBITAK, 2010). He completed another postdoc in the field of nanotechnology at the Tamkang University Taipei (20132014) sponsored by National Science Council now M/o Science and Technology, Taiwan (R.O.C.). 
Presently, he is working as Assistant Professor on tenure track at COMSATS University Islamabad (previously known as COMSATS Institute of Information Technology), Islamabad, Pakistan (since May 2008) and prior to that worked as assistant director/deputy director at M/o Science \& Technology (Pakistan Council of Renewable Energy Technologies, Islamabad, 2000-2008). He was invited by Institute for Materials Research, Tohoku University, Japan to deliver scientific talk. He gave several scientific talks in various countries. His core area of research includes materials science, physics \& nanotechnology. He was also offered the merit scholarship for the PhD study by the Higher Education Commission, Government of Pakistan, but he did not avail himself of the opportunity. He also earned Diploma (in English language) and Certificate (in Japanese language) in 2000 and 2001 respectively, in part-time from the National University of Modern Languages, Islamabad. He is the author of several articles

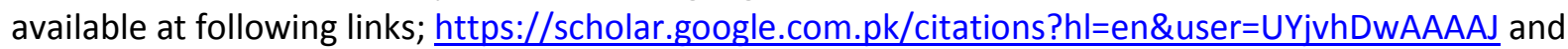
https://www.researchgate.net/profile/Mubarak Ali5 